\title{
Reduced Pyramidal Cell Somal Volume in Auditory Association Cortex of Subjects with Schizophrenia
}

\author{
Robert A Sweet', Joseph N Pierri', Sungyoung Auh ${ }^{3}$, Allan R Sampson ${ }^{3}$ and David A Lewis*, 1,2 \\ 'Department of Psychiatry, University of Pittsburgh, Pittsburgh, PA, USA; ${ }^{2}$ Department of Neuroscience, University of Pittsburgh, Pittsburgh, PA, \\ USA; ${ }^{3}$ Department of Statistics, University of Pittsburgh, Pittsburgh, PA, USA
}

\begin{abstract}
Subjects with schizophrenia have decreased gray matter volume of auditory association cortex in structural imaging studies, and exhibit deficits in auditory sensory memory processes subserved by this region. In dorsal prefrontal cortex (dPFC), similar in vivo observations of reduced regional volume and working memory deficits in subjects with schizophrenia have been related to reduced somal volume of deep layer 3 pyramidal cells. We hypothesized that deep layer 3 pyramidal cell somal volume would also be reduced in auditory association cortex (BA42) in schizophrenia. We used the nucleator to estimate the somal volume of pyramidal neurons in deep layer 3 of BA42 in 18 subjects with schizophrenia, each of whom was matched to one normal comparison subject for gender, age, and postmortem interval. For all subject pairs, somal volume of pyramidal neurons in deep layer 3 of dPFC (BA9) had previously been determined. In BA42, somal volume was reduced by $13.1 \%$ in schizophrenic subjects $(p=0.03)$. Reductions in somal volume were not associated with the history of antipsychotic use, alcohol dependence, schizoaffective disorder, or death by suicide. The percent change in somal volume within-subject pairs was highly correlated between BA42 and BA9 $(r=0.67, p=0.002)$. Deep layer 3 pyramidal cell somal volume is reduced in BA42 of subjects with schizophrenia. This reduction may contribute to impairment in auditory function. The correlated reductions of somal volume in BA42 and BA9 suggest that a common factor may affect deep layer 3 pyramidal cells in both regions.

Neuropsychopharmacology (2003) 28, 599-609. doi:10.1038/sj.npp. 1300120
\end{abstract}

Keywords: auditory cortex; neuron volume; pyramidal cells; schizophrenia

\section{INTRODUCTION}

Several lines of evidence indicate abnormalities of auditory association cortex in subjects with schizophrenia. Functional and electrophysiological testing of audition reveal deficits in the precision of auditory sensory memory in subjects with schizophrenia (Strous et al, 1995; Javitt et al, 1997, 2000a; Rabinowicz et al, 2000). Although the data are limited, in nonhuman primates precision of auditory sensory memory appears to be dependent on bilaterally intact auditory association cortex (Dewson III et al, 1970; Cowey and Dewson, 1972; Iverson and Mishkin, 1973; Cowey and Weiskrantz, 1976; Colombo et al, 1990). In contrast, lesions of primary auditory cortex are predominantly associated with reduced auditory acuity (Heffner and Heffner, 1989; Heffner, 1997), aspects of audition that are not prominently impaired in subjects with schizophrenia (Mathew et al, 1991).

\footnotetext{
*Correspondence: Dr DA Lewis, University of Pittsburgh, 38 I I O'Hara Street, WI 650 BST, Pittsburgh, PA 15213, USA, Tel: + | 4126243934 , Fax: + 4126249910, E-mail: lewisda@msx.upmc.edu

Received 4 June 2002; revised 23 September 2002; accepted 22 November 2002

Online publication: 3 December 2002 at http://www.acnp.org/ Citations/Npp 120302436
}

The auditory association cortex (Brodmann Area 42, BA42) lies on the dorsal bank of the superior temporal gyrus (STG), immediately posterolateral to the primary auditory cortex, which is located in Heschl's gyrus (Figure 1) (Garey, 1999). More than 20 MRI studies have examined cortical gray matter volume of the STG in subjects with schizophrenia (McCarley et al, 1999; Goldstein et al, 1999; Sanfilpo et al, 2000; Mathalon et al, 2001; Sigmundsson et al, 2001; Hirayasu et al, 2001). Of these studies, $80 \%$ have found reduced gray matter volume in subjects with schizophrenia, a more consistent change than in any other cortical region (McCarley et al, 1999). Gray matter reductions in STG are present in subjects with schizophrenia at the time of their first episode, but are not found in bipolar disorder subjects with manic psychosis and are not prominent in alcohol-dependent subjects (Hirayasu et al, 1998, 2001; Sullivan et al, 1998). Although most MRI studies have included Heschl's gyrus within the measured volume of STG, in two studies to measure separately gray matter volume of Heschl's gyrus, it was found to be less affected than adjacent STG (Barta et al, 1997; Hirayasu et al, 2001).

The alterations in cells and neurocircuitry that underlie reduced gray matter volume in STG of subjects with schizophrenia are not known. In dorsal prefrontal cortex 

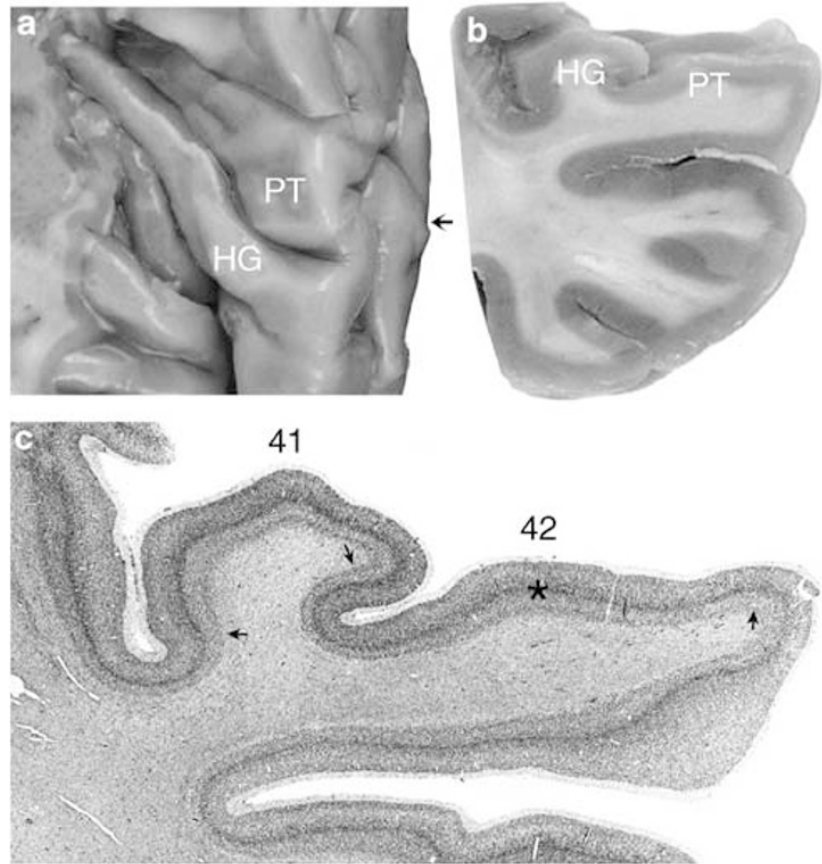

Figure I (a) Indicates a photograph of the dorsal surface of the superior region of the left temporal lobe of a comparison subject. The brain is oriented with rostral down, caudal up, and medial to the left. Heschl's gyrus $(\mathrm{HG})$, which contains the primary auditory cortex (BA4I), is indicated. Immediately posterolateral to $\mathrm{HG}$ on the STG is the planum temporale (PT), which contains the auditory association cortex BA42. The arrow at the lateral margin of the STG indicates the rostral-caudal level of the tissue block seen in (b). (c) Enlarged view of a section from the block in (b), stained with thionin for Nissl substance. Arrows in white matter identify the medial and lateral boundaries of the primary auditory cortex (BA4I) and auditory association cortex (BA42). The asterisk indicates the approximate location of the photomicrograph presented in Figure 2.

(dPFC), another region where MRI studies have found reduced gray matter volume (McCarley et al, 1999), postmortem studies have identified reductions in pyramidal cell somal volume in subjects with schizophrenia. Rajkowska et al (1998) described a decrease in the mean somal size of all layer 3 neurons in BA9. Consistent with that report, we recently found that the mean somal volume of deep layer 3 pyramidal cells was reduced in BA9 in a study of 28 matched pairs of subjects with schizophrenia and comparison subjects (Pierri et al, 2001). Reductions in pyramidal cell somal volume directly contribute to reduced gray matter volume. In addition, because neuronal size is correlated with the extent of a neuron's dendritic arbor, reduced somal volume may also indirectly contribute to reduced gray matter volume because of reduced neuropil volume (Jacobs et al, 1997; Pierri et al, 2001).

We therefore examined deep layer 3 pyramidal cell somal volume in auditory association cortex (BA42) of subjects with schizophrenia and comparison subjects. To compare findings in this region with our earlier findings in BA9, all subject pairs previously studied in BA9, for whom auditory cortex tissue was available, were studied. We sought to answer two questions: (1) Is the mean somal volume of deep layer 3 pyramidal cells reduced in BA42 in subjects with schizophrenia? (2) How are reductions in somal volume of deep layer 3 pyramidal cells in BA42 and BA9 related within the same pairs of subjects?

\section{METHODS}

\section{Subjects}

Of the 28 subject pairs included in our study of BA9 (Pierri et al, 2001), auditory cortex tissue was available for 18 pairs (Table 1). Brain specimens had been obtained from these subjects during autopsies conducted at the Allegheny County Coroner's Office, after receiving consent from the next of kin using a mechanism approved by the Institutional Review Board of the University of Pittsburgh. Neuropathological examinations revealed no abnormalities in any of the subjects except for the following. Thioflavin-S staining revealed a few neuritic plaques in one normal subject (pair 2), but he did not meet either clinical or neuropathological criteria for Alzheimer's disease (Mirra et $a l$, 1991). One subject with schizophrenia died of a cerebrovascular event (pair 10, intracerebral hemorrhage in the right temporal lobe). An independent panel of experienced clinicians made consensus DSM-III-R diagnoses as described previously (Glantz and Lewis, 2000).

The subjects diagnosed with schizophrenia $(n=13)$ or schizoaffective disorder $(n=5$, Table 1$)$ had each been previously matched to one normal comparison subject for gender, age, and post-mortem interval (PMI) (Pierri et al, 2001). In the 18 pairs available for this report, the mean age $( \pm$ SD) did not differ between subjects with schizophrenia $(47.3 \pm 6.8$ yeas $)$ and comparison subjects $(47.2 \pm 8.2$ years). Mean ( \pm SD) PMI was significantly longer in subjects with schizophrenia than in comparison subjects $\left(15.4 \pm 6.3 v s 13.6 \pm 5.1 \mathrm{~h}, T_{17}=2.3, p=0.03\right)$. The mean $( \pm$ SD) age of onset and duration of illness were $25.2 \pm 8.8$ and $22.4 \pm 8.1$ years, respectively, for the subjects with schizophrenia. Five of the subjects with schizophrenia met diagnostic criteria for alcohol dependence at the time of death, and one other subject with schizophrenia had a lifetime history of alcohol dependence. Toxicology examinations were positive for plasma alcohol in two subjects with schizophrenia (pairs $12(0.12 \%)$ and $8(0.09 \%))$ and in one comparison subject (pair $3(0.01 \%)$ ). Three subjects with schizophrenia had been off antipsychotic medication for at least one month prior to death, one of whom (pair 5) had never received treatment.

\section{Tissue Preparation}

The left hemisphere of each brain was blocked coronally at $1.0-2.0 \mathrm{~cm}$ intervals, immersed in ice-cold $4 \%$ paraformaldehyde in phosphate buffer for $48 \mathrm{~h}$, washed in a graded series of sucrose solutions, and stored in an antifreeze solution at $-30^{\circ} \mathrm{C}$. Tissue storage time did not differ between the subjects with schizophrenia and comparison subjects (78.4 \pm 26.4 vs $76.8 \pm 28.2$ months, respectively). Tissue blocks located caudal to the level of the mammillary bodies and rostral to the crux of the fornix (Shenton $e t$ al, 1992), and matched on rostral-caudal level within pairs, were identified (Figure 1). Coronal cryostat sections $(40 \mu \mathrm{m})$ were cut throughout the full block thickness. Every 10th section selected using a random starting number was immersed for $24 \mathrm{~h}$ in cold $4 \%$ buffered paraformaldehyde, rinsed in buffer, and mounted to gelatin-subbed slides. After drying overnight, sections were stained for Nissl substance with thionin. 
Table I Subject Characteristics

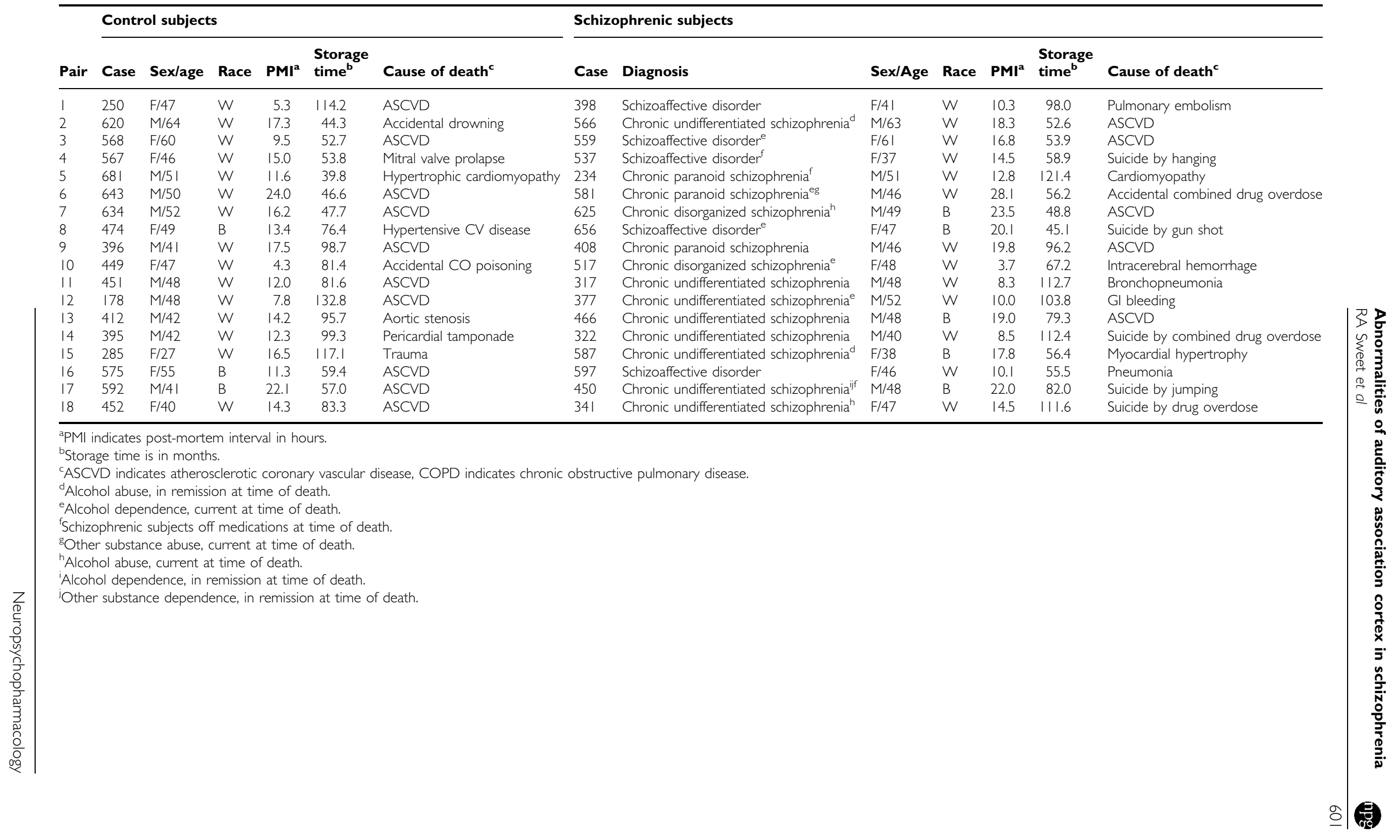




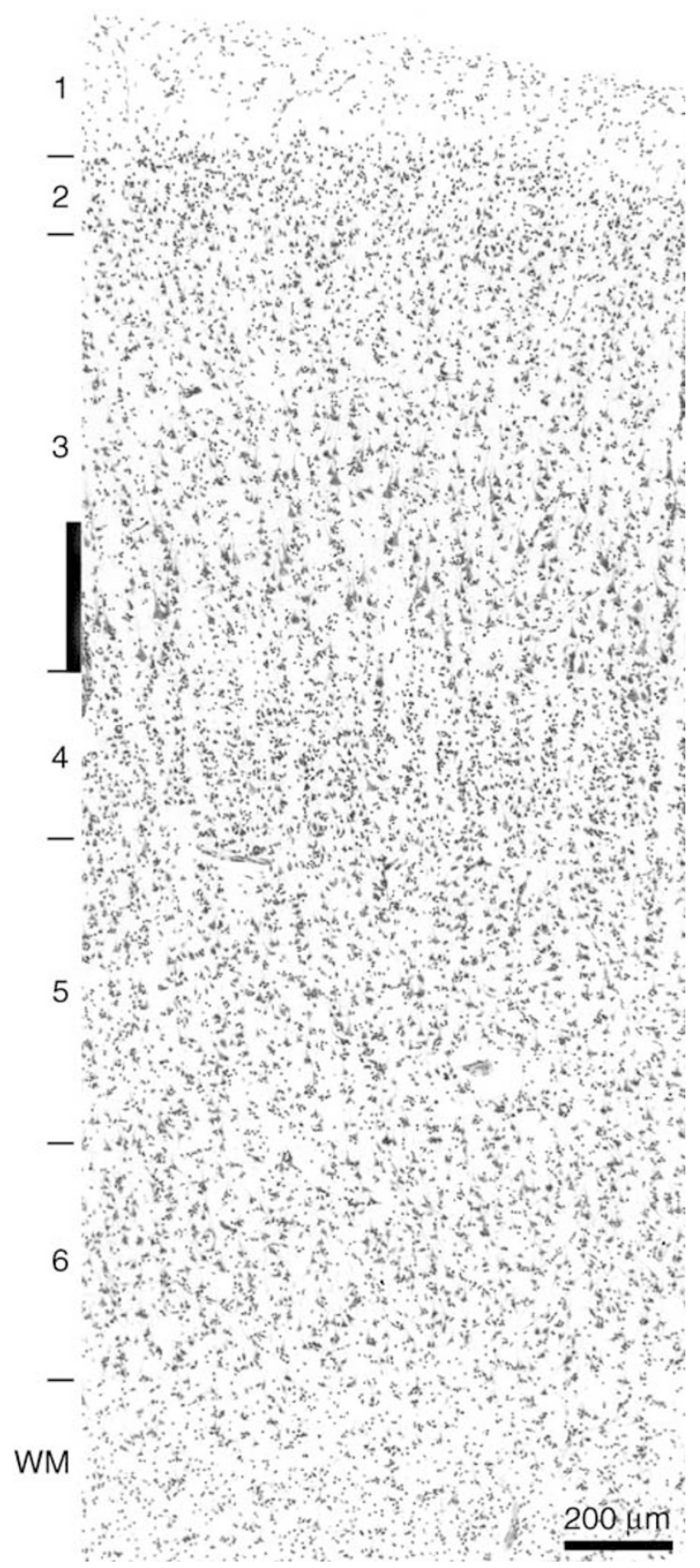

Figure 2 Photomicrograph of BA42 taken from the section shown in Figure Ic. The distinct features of BA42 are evident: granular layers 2 and 4 , well-developed layer 3 with distinct columnar arrangement of pyramids, large deep layer 3 pyramids, a relatively hypocellular layer 5, and distinct layer 6. Bracket at right border indicates deep layer 3.

BA42 was identified by its location lateral to the primary auditory cortex and the following cytoarchitectonic features: prominent layers 2 and 4, pyramids arranged in columns in layer 3 , reduced layer 3 cellularity in comparison with primary auditory cortex, large deep layer 3 pyramids, a hypocellular layer 5, and distinct layer 6 (Figure 2). BA42 was differentiated from the more posterolateral BA22, which demonstrates a less well-developed layer 4 , increased cell density and cell size in layer 5 , and branching columns in layer 6 (Galaburda and Sanides,
1980). For each subject, all Nissl-stained sections were reviewed, and the most rostral section containing BA42 cut in an orientation perpendicular to the pial surface was identified. This section and two additional sections, 1600 and $3200 \mu \mathrm{m}$ caudal to the initial section and containing BA42 cut with the vertical axes of cells in an orientation perpendicular to the pial surface, were selected for analysis. In one subject, the section $1600 \mu \mathrm{m}$ caudal did not contain BA42 in a perpendicular orientation, so the section $2000 \mu \mathrm{m}$ caudal to the initial section was substituted. All tissue sections were coded to conceal subject number and diagnosis.

\section{Measurement of Somal Size}

Quantification was performed without knowledge of diagnosis by one investigator (RAS). A Zeiss Axioplan microscope equipped with Stereo Investigator software (MicroBrightField, Inc., Colchester, VT) was used to identify BA42 at low magnification $(\times 50)$. The borders of layers 2, 3 and 4 were located (Figure 2), and the thickness of layer 3 was determined. In order to evaluate a population of neurons comparable to those previously studied in BA9 (Pierri et al, 2001), a similar contour outlining the deepest of layer 3 was drawn (Figures 2 and 3). There was no significant difference between groups in mean $( \pm$ SD) layer 3 thickness/section $(825 \pm 138 \mu \mathrm{m}$ for the comparison subjects and $854 \pm 111 \mu \mathrm{m}$ for the subjects with schizophrenia, $\mathrm{F}_{1,106}=1.4, p=0.2$ ) or in mean $( \pm \mathrm{SD}$ ) contour area/section $\left(1.04 \pm 0.51 \times 10^{6} \mu^{2}\right.$ for the comparison subjects and $0.89 \pm .40 \times 10^{6} \mu \mathrm{m}^{2}$ for the subjects with schizophrenia, $\left.\mathrm{F}_{1,106}=3.1, p=0.08\right)$. Cell measurements were obtained using a 1.4 numerical aperture, $\times 100$, oil immersion objective, with visualization on a computer monitor at a final magnification of $\times 2450$. To randomly sample cells, we used the optical fractionator (Gundersen et al, 1988) probe of the Stereo Investigator software, which systematically and randomly placed sampling boxes throughout the region of interest (Figure 3). Each box was $90 \times 60 \times 8 \mu \mathrm{m}$ in the $x, y$, and $z$ directions, respectively. At each sampling site, sampling was begun $2 \mu \mathrm{m}$ below the section surface. The final mean $( \pm$ SD) section thickness did not differ between groups $(12.7 \pm 1.5 \mu \mathrm{m}$ for the comparison subjects and $12.2 \pm 0.7 \mu \mathrm{m}$ for the subjects with schizophrenia, $\mathrm{F}_{1,34}=1.3, p=0.3$ ).

To estimate somal volume, we used the nucleator method (Gundersen, 1988) as implemented in the Stereo Investigator software. Three-dimensional objects have as fundamental properties a volume and a surface area, but do not have a unique cross-sectional area. The nucleator probe, although taking measurements in a single plane, is advantageous in comparison with measurement of the cross-sectional area because it is an unbiased estimator of the volume of an object. This estimate is independent of the object's shape, under the following assumptions (as applied in the current study): the measurements are taken in a focal plane sufficiently narrow to avoid overprojection; the neurons measured are isotropically oriented with regard to the focal plane; and tissue distortion during histological processing does not alter the neuronal dimensions in the $x$ and $y$-axis. For each neuron, the nucleolus was used as the cell's uniquely associated reference point. To estimate somal 
a

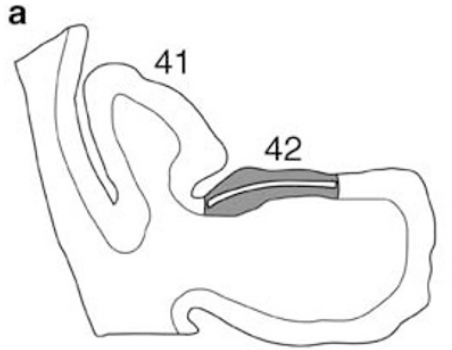

b

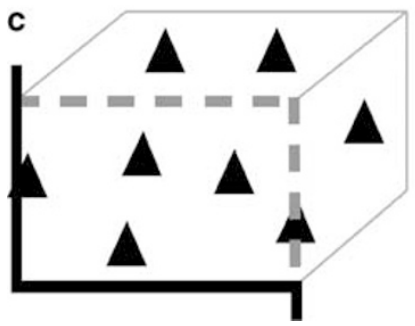

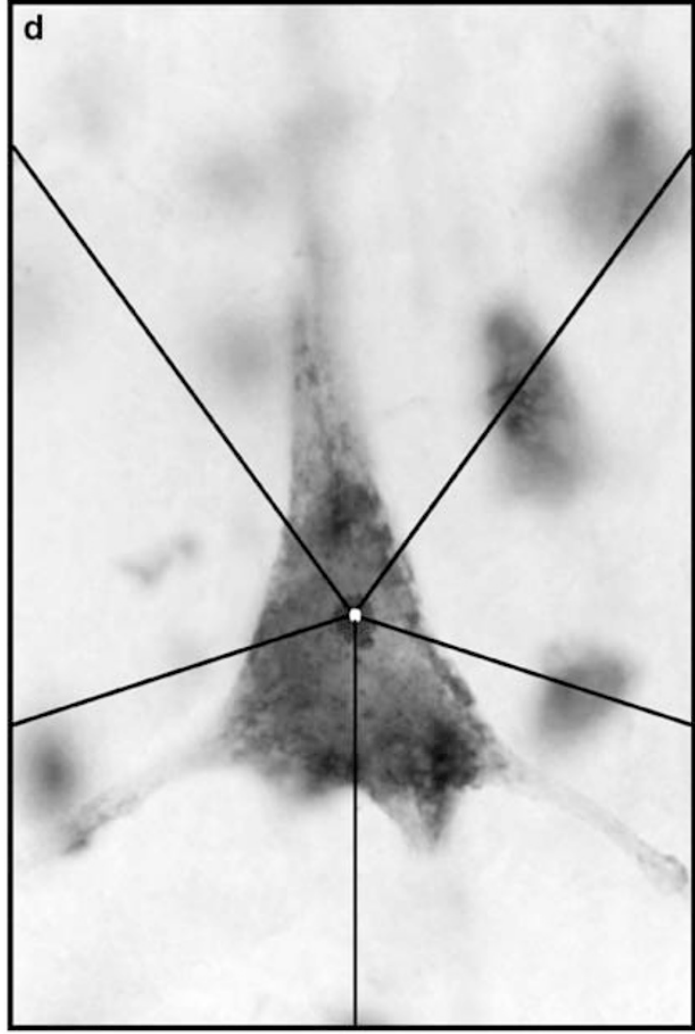

Figure 3 Schematic summary of the method used to determine somal volume. (a) Schematic of a coronal section through Heschl's gyrus and PT, showing BA42 (shaded). A contour was placed in the lower I/3 of layer 3. (b) Representative sampling grid overlaying the contour in (a). Within the grid is a systematic random distribution of three-dimensional counting bricks. (c) Counting brick. Heavy dashed and solid lines indicate inclusion and exclusion boundaries, respectively. (d) Nucleated Nissl-stained pyramidal cell. The intersection of each isotropic ray with the somal margin is used to generate an estimate of somal volume.

size, the operator clicked on the nucleolus, which brought up a set of five, two-dimensional isotropic random rays (Figure 3). The operator clicked on each ray where it intersected the boundaries of the soma. Volume was calculated as: volume $=4 / 3 \pi \hat{I}_{n}{ }^{3}$, where $\hat{I}$ equals the mean segment length from the nucleolus to the cell boundary and $n$ equals the number of segments, in this case, 5 .

Only cells within the three-dimensional sampling box meeting the following criteria for pyramidal neurons were measured: (1) an identifiable nucleolus, (2) an abundance of Nissl-stained cytoplasm, (3) a vertical apical dendrite, and (4) a triangular shape (Figure 4$)$. The mean $( \pm S D)$ number of neurons measured for each subject did not differ between the comparison subjects and the subjects with schizophrenia $\left(202 \pm 31\right.$ vs $196 \pm 35$, respectively, $\left.\mathrm{F}_{1,34}=0.2, p=0.6\right)$. The mean $( \pm S D)$ coefficients of error for somal volume were $8.1 \pm 1.5 \%$ and $8.8 \pm 2.3 \%$ for the comparison subjects and the subjects with schizophrenia, respectively.

\section{Statistical Methods}

Since both individual and group somal volume distributions for all measured neurons in each diagnostic group showed a skew toward the larger somal sizes, all single neuron volume estimates were transformed using a natural log function (denoted by $\ln$ ) prior to statistical testing (Pierri et al, 2001). For each subject, estimates of $\ln$ somal volume were averaged over the neurons within each of the three sections.
These averages were treated as three correlated observations. To examine a main effect of diagnosis, multivariate analysis of covariance (MANCOVA) models, all assuming a compound symmetric covariance structure were employed (Neter et al, 1996). The analysis reflected possible correlations of the three observations within a given subject. The dependent variables were the three averaged $\ln$ somal volumes for each subject. The main effect of diagnostic group was evaluated in two models in order to assess the robustness of the diagnostic effect: (1) With pair as a blocking factor and tissue storage time as a covariate; and (2) with gender, age, PMI, and tissue storage time entered as covariates and with subject pairings ignored. Preliminary to the two primary MANCOVA analyses for diagnostic group effect, MANCOVA analyses were undertaken to assess if there were possible slide order (rostral to caudal) or handedness effects. The intention was to not include slide order or handedness in the primary analysis if significant effects were not observed in the preliminary analyses. In the 28 subjects for whom handedness information was available, handedness was evaluated using the following MANCOVA model: diagnosis, age, gender, PMI, tissue storage time, handedness, and handedness ${ }^{*}$ diagnosis. To examine slide-order effect, the MANCOVA model included: pair, slide order, and slide order*diagnosis.

Analyses were implemented in SAS PROC Mixed (Littell et al, 1996). Summary descriptions of somal volume were obtained by backtransforming the summary statistics of $\ln$ 


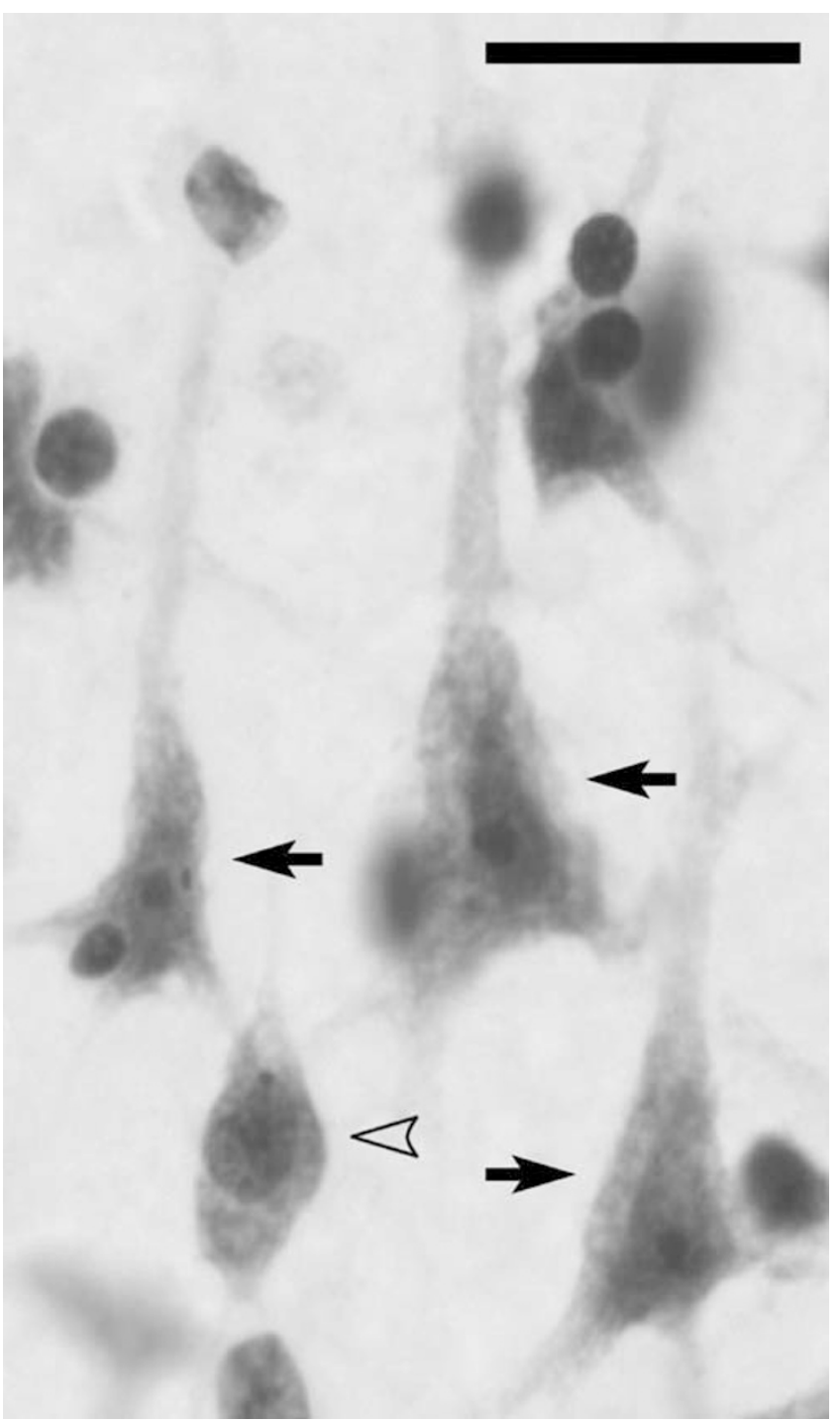

Figure 4 Photomicrograph of Nissl-stained neurons in deep layer 3. Filled arrows indicate neurons meeting the study criteria for selection of pyramidal cells for somal volume determination. A neuron not meeting the study criteria for selection is also indicated by an open arrowhead. (Photomicrograph is a $z$-axis montage, scale bar $=20 \mu \mathrm{m}$.)

somal volume. Backtransformation yields geometric means that are estimates of median somal volume (Johnson et al, 1994). The distributions of somal size for the two diagnostic groups are described using 25th and 75th percentiles, obtained through backtransformation.

Examination of the effects of antipsychotic use, alcohol dependence, suicide, and schizoaffective disorder used ANOVA. For these analyses, the three observations of $\ln$ somal volume in each subject were collapsed into a single mean value, and then backtransformed to yield a geometric mean value. The percent change relative to control within each pair was computed as the dependent variable. This resulted in 18 observations. The effects of these clinical variables were examined using independent samples $t$-tests (assuming common variances) with each variable as a grouping factor.

To examine changes in cell densities between diagnostic groups, arbitrary cutoff values were used to create four somal size categories (Pierri et al, 2001). An overall density including all pyramidal cells and the density for each of these four groups was calculated for each section. The densities for each of the categories were independently analyzed to examine the diagnostic group effect using a MANCOVA model with diagnosis, pair, and tissue storage time.

Tests of correlations between BA42 and BA9 used Pearson's $r$. To examine whether the magnitude of change in ln somal volume differed between BA42 and BA9 a MANCOVA model used average ln somal volume in the two regions as a repeated measure, with diagnosis, pair, and tissue storage time as covariates.

All statistical tests were conducted with an $\alpha$ level $=0.05$. When appropriate the Bonferroni correction was used to control for multiple comparisons.

\section{RESULTS}

The distributions of all pyramidal cell somal volumes in subjects with schizophrenia and comparison subjects are presented in Figure 5. Ln somal volume was significantly reduced in the subjects with schizophrenia in Model 1 $\left(\mathrm{F}_{1,16}=5.9, p=0.03\right)$. Geometric mean somal volume of the subjects with schizophrenia was $1584.3 \mu^{3}$ (25th percentile: $1337.1 \mu^{3}$; 75 th percentile: $1877.2 \mu \mathrm{m}^{3}$ ) as compared to $1813.7 \mu^{3}$ (25th percentile: $1562.5 \mu \mathrm{m}^{3}$; 75 th percentile: $2105.4 \mu^{3}$ ) in the comparison subjects. Model 2 confirmed the significant effect of diagnosis on ln somal volume $\left(\mathrm{F}_{1,30}=4.5, p=0.04\right)$, without a significant effect upon $\ln$ somal volume because of subject age $\left(\mathrm{F}_{1,30}=2.0, p=0.2\right)$, gender $\left(\mathrm{F}_{1,30}=0.8, p=0.4\right)$, PMI $\left(\mathrm{F}_{1,30}=0.1, p=0.7\right)$, or tissue storage time $\left(F_{1,30}=2.0, p=0.2\right)$. Ln somal volume was not significantly associated with subject handedness $\left(\mathrm{F}_{1,20}=2.8, \quad p=0.1\right)$, handedness ${ }^{\star}$ diagnosis $\left(\mathrm{F}_{1,20}=0.2\right.$, $p=0.7)$, slide order $\left(\mathrm{F}_{2,68}=0.9, p=0.4\right)$, or slide order ${ }^{\star}$ diagnosis $\left(\mathrm{F}_{2,68}=1.0, p=0.4\right)$. Somal volume was reduced in the subject with schizophrenia in $11 / 18$ subject pairs. Neither antipsychotic use at the time of death, comorbid alcohol dependence, nor death by suicide were significantly

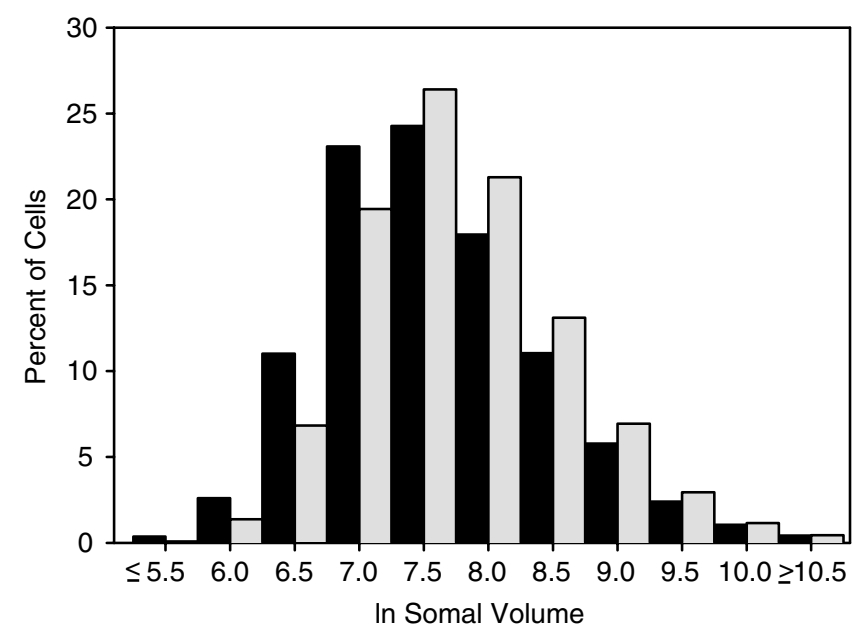

Figure 5 Pyramidal cell somal volume distributions in BA42 for subjects with schizophrenia (black bars) and comparison subjects (gray bars). Bar height is presented as a percent of the total number of pyramidal cells measured within each group. 
associated with the within-subject pair differences in somal volume (Table 2). Although the magnitude of somal volume reduction was greater in subjects diagnosed with schizoaffective disorder than with schizophrenia, this difference was not significant.

As shown in Table 3, the overall pyramidal cell density did not differ between subject groups. Modest, nonsignificant decreases in the density of medium, large, and extralarge pyramidal cells in the subjects with schizophrenia were offset by an increase in the density of small cells.

We next examined the relation between pyramidal cell somal volumes in BA42 and in BA9. Averaged ln somal volume in these two regions was significantly correlated in comparison subjects $(r=0.52, p=0.03)$, whereas in the subjects with schizophrenia, the degree of correlation was smaller and did not reach statistical significance $(r=0.32$, $p=0.2$ ). As expected from previous qualitative cytoarchitectonic comparisons, the geometric mean somal volumes were smaller in BA42 than in BA9 in both subject groups (Figure 6a). The percent reduction in the geometric mean somal volume was $13.1 \%$ in BA42 as compared to $9.4 \%$ in BA9, without a significant interaction of diagnosis by brain region $\left(\mathrm{F}_{1,33}=0.3, p=0.6\right)$. The within pair percent changes in geometric mean somal volumes were significantly correlated between regions BA9 and BA42 $(r=0.67$, $p=0.002$, Figure $6 \mathrm{~b}$ ).

Table 2 Effect of Clinical Variables on the Percent Change, within Matched Pairs of Subjects, of Deep Layer 3 Pyramidal Cell Geometric Mean Somal Volumes

\begin{tabular}{|c|c|c|c|c|}
\hline & $\mathbf{N}$ & $\begin{array}{l}\text { Percent } \\
\text { change } \pm \text { SD }\end{array}$ & $|t|$ & $p$ \\
\hline $\begin{array}{l}\text { Antipsychotic medication use } \\
\text { at death }\end{array}$ & & & 0.1 & 0.9 \\
\hline Off & 3 & $-8.3 \pm 29$ & & \\
\hline On & 15 & $-10.1 \pm 23$ & & \\
\hline Alcohol dependence & & & 1.1 & 0.3 \\
\hline No & 12 & $-13.2 \pm 24$ & & \\
\hline Yes & 6 & $-2.2 \pm 20$ & & \\
\hline Suicide & & & 1.3 & 0.2 \\
\hline No & 13 & $-\mid 4.3 \pm 21$ & & \\
\hline Yes & 5 & $1.6 \pm 27$ & & \\
\hline Schizoaffective disorder & & & 1.0 & 0.3 \\
\hline No & 13 & $-6.5 \pm 25$ & & \\
\hline Yes & 5 & $-18.7 \pm 15$ & & \\
\hline
\end{tabular}

\section{DISCUSSION}

Mean deep layer 3 pyramidal cell somal volume in auditory association cortex, BA42, was reduced by $13.1 \%$ in subjects with schizophrenia. This reduction did not appear to be attributable to other characteristics such as age, gender, handedness, antipsychotic use at the time of death, comorbid alcohol use disorder, or death by suicide, nor
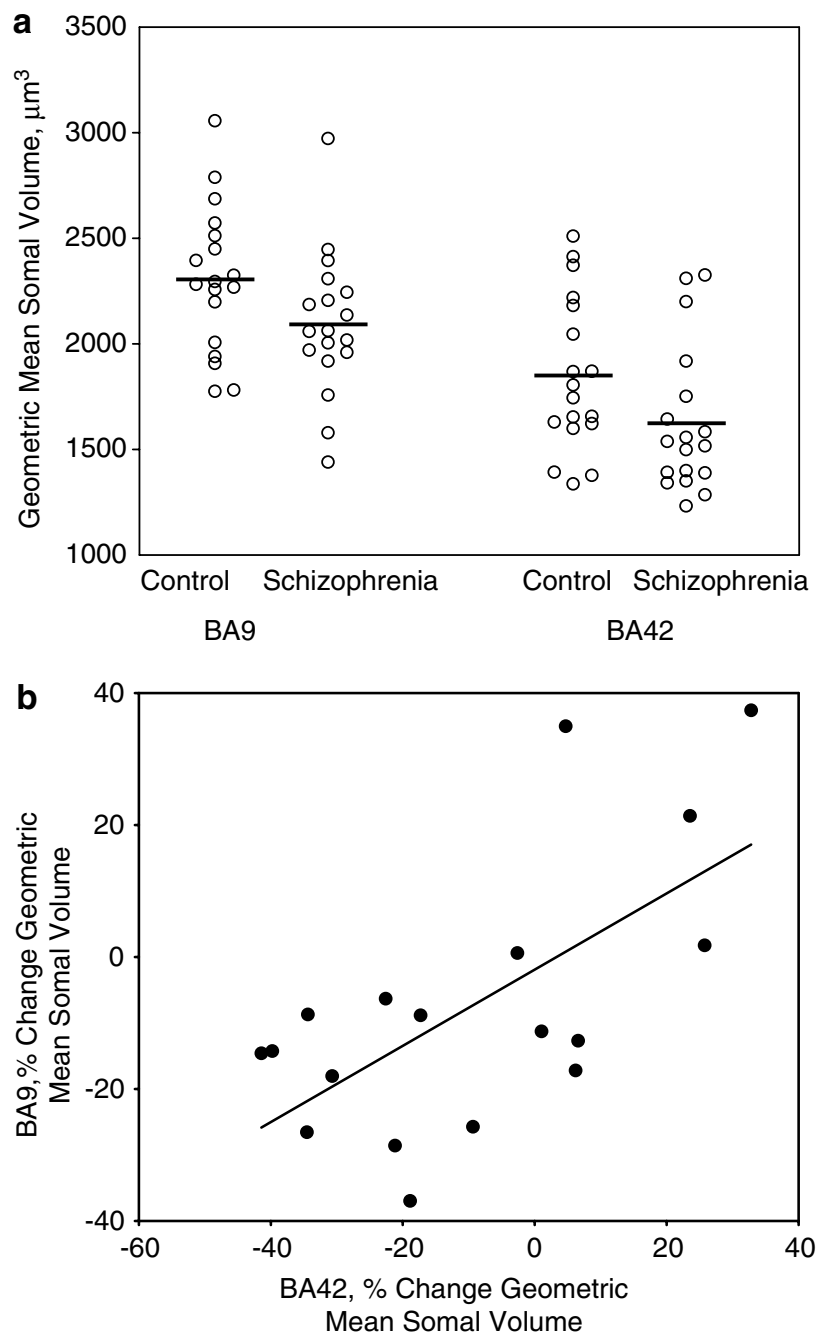

Figure 6 (a) Comparison of geometric mean pyramidal cell somal volume in BA42 and BA9 in individual subjects. The magnitude of reduction of mean pyramidal cell somal volume was not different between regions. (b) The relation between differences in mean pyramidal cell somal volume within-subject pairs in BA42 and BA9. The changes were highly correlated between regions $(r=0.67, p=0.002)$

Table 3 Pyramidal Cell Mean Densities $( \pm S D)$ in Deep Layer 3 of BA42 of Subjects with Schizophrenia and Comparison Subjects

\begin{tabular}{llcccl}
\hline $\begin{array}{l}\text { Pyramidal } \\
\text { cell class }\end{array}$ & $\begin{array}{l}\text { Pyramidal cell } \\
\text { size }\left(\boldsymbol{\mu m}^{\mathbf{3}}\right)\end{array}$ & $\begin{array}{l}\text { Comparison } \\
\text { subjects }\end{array}$ & Schizophrenia & $\mathbf{F}_{\mathbf{I}, \mathbf{1 6}}$ & $\begin{array}{l}\text { Bonferroni } \\
\text {-adjusted } \boldsymbol{p}\end{array}$ \\
\hline Small & $<1000$ & $9.6 \pm 3.9$ & $12.2 \pm 5.1$ & 3.6 & 0.3 \\
Medium & $1001-3000$ & $20.6 \pm 3.2$ & $17.4 \pm 3.5$ & 7.7 & 0.054 \\
Large & $3001-5000$ & $5.3 \pm 2.0$ & $4.2 \pm 1.0$ & 5.4 & 0.1 \\
Extralarge & $>5000$ & $4.4 \pm 1.9$ & $3.4 \pm 1.6$ & 4.6 & 0.2 \\
All & & $39.8 \pm 4.9$ & $37.2 \pm 6.4$ & 1.7 & 0.2 \\
\hline
\end{tabular}


did the magnitude of somal volume reduction differ significantly between subjects with schizophrenia and subjects with schizoaffective disorder. There was no significant change in the overall density of pyramidal cells. The reduction in mean deep layer 3 pyramidal cell somal volume was not significantly greater in BA42 than in BA9. Within-subject pairs, the magnitude of reduced mean pyramidal cell somal volume was highly correlated between BA9 and BA42.

The finding of reduced pyramidal cell somal volume in deep layer 3 of BA42 adds to a growing body of evidence that pyramidal cell and/or neuronal size is reduced in subjects with schizophrenia. Reduced neuronal size has been reported in hippocampal subfields and in layer 3 of BA9, but not in other layers of BA9, nor in motor or visual cortices (Benes et al, 1991; Arnold et al, 1995; Zaidel et al, 1997; Rajkowska et al, 1998; Pierri et al, 2001). An important constraint in the interpretation of each of these findings is that reduced neuronal somal volume could result from either a reduction in size of neurons without a change in total neuron number, a reduction in the number of large neurons, or an increase in the number of small neurons. Visual inspection of Figure 5 suggests a relatively uniform reduction of somal volume in the present study, rather than a change in the number of neurons in a specific cell size class. Consistent with this interpretation, we found that in conjunction with modest decreases in densities of medium, large, and extralarge cells in BA42, there was an increased density of small cells that was similar in magnitude. Overall, there was no significant difference in the density of deep layer 3 pyramidal cells between comparison subjects and subjects with schizophrenia. Although Rajkowska et al (1998) identified a reduced density of extralarge neurons in layer 3c of BA9, a corresponding, although nonsignificant, increase in the density of small and medium size neurons was also present. Extrapolation from measures of cell density to conclusions about cell number, however, should be viewed cautiously in the absence of independent measurement of the complete reference volume of the region (Mendis-Handagama and Ewing, 1990). Indeed, if the reductions in STG gray matter volume found in MRI studies of subjects with schizophrenia are present in deep layer 3 of BA42, then the lack of change in cell density would indicate a reduced total number of neurons. In the one study to use unbiased stereologic methods to estimate the total neuron number in the neocortex of subjects with schizophrenia, no significant change in cell number was found in PFC or temporal lobe (Pakkenberg, 1993). However, that study may not have had the sensitivity to detect a reduction in the number of cells of a specific size class, within a single cortical layer, in a specific cytoarchitectonic region. Final resolution of this question will require the estimation of the total cell number in deep layer 3 of BA42.

The observation of reduced mean pyramidal cell somal volume in BA42, regardless of whether resulting from smaller or fewer cells, is consistent with both MRI and postmortem findings of reduced gray matter volume of the STG of subjects with schizophrenia (Highley et al, 1999; Falkai et al, 1995; Vogeley et al, 1998; McCarley et al, 1999). However, the smaller mean pyramidal cell size observed in deep layer 3 of BA42 is unlikely to represent the only source contributing to the observation of reduced gray matter volume in this region. Pyramidal cell somal volume has been found to correlate with the extent of dendritic arborization and number of dendritic spines (Jacobs et al, 1997), both of which contribute to gray matter volume. In layer 3 of dPFC, all three of these structural markers appear to be diminished in concert in subjects with schizophrenia (Glantz and Lewis, 2000; Pierri et al, 2001). Whether deficits in dendritic arborization also contribute to gray matter volume loss in BA42 is not known.

It is important to consider whether the correlation in somal volume reduction across brain regions might not result from a shared 'vulnerability', but rather from a shared comorbid condition or from treatment with antipsychotic medications. Structural imaging studies have not found reduced STG gray matter volume in bipolar disorder subjects with manic psychosis (Hirayasu et al, 2001). Nor do subjects with chronic alcohol use disorders demonstrate prominent loss of STG gray matter volume (Sullivan et al, 1998). Consistent with the in vivo findings, we did not find an association of reduced pyramidal cell somal volume in BA42 with a diagnosis of alcohol dependence. Arguing further against an effect of comorbid alcohol dependence on somal volume, the absolute magnitude of pyramidal cell somal volume reduction was smaller in those subjects with comorbid alcohol dependence than in subjects without alcohol dependence.

We did not observe an association of reduced pyramidal cell somal volume with antipsychotic use at the time of death in BA42, although only one subject had no lifetime history of antipsychotic use. Nor did we previously observe an association between antipsychotic use at the time of death and mean pyramidal cell somal volume in deep layer 3 of BA9 (Pierri et al, 2001). Moreover, in vivo structural imaging studies have found that reduced STG gray matter volume is present in first-episode subjects, prior to longterm exposure to antipsychotic medication (Hirayasu et al, 1998; Hirayasu et al, 2001). Antipsychotic treatment, however, has been shown to reduce dendritic and spinerelated protein expression in multiple brain regions of nonhuman primate (Lidow et al, 2001). Whether antipsychotic medication treatment also contributes to reduced pyramidal cell somal volume has not been evaluated in a nonhuman primate model.

Whether any systematic methodologic bias may have contributed to the observations of reduced pyramidal cell somal volume in subjects with schizophrenia in the current, or in prior studies (Benes et al, 1991; Arnold et al, 1995; Zaidel et al, 1997; Rajkowska et al, 1998; Pierri et al, 2001) also needs to be considered. We examined pyramidal cells in the deepest $1 / 3$ rd of layer 3. An advantage of this definition is that it does not depend on recognition of the subtle cytoarchitectonic boundary between sublayers $3 \mathrm{~b}$ and $3 \mathrm{c}$. It is possible, however, that the reduced pyramidal cell somal volume we observed could result from thinning of layer $3 c$ in subjects with schizophrenia, with resulting inclusion of the smaller layer $3 b$ cells in our sampling contours. Arguing against this possibility, the thickness of layer 3 did not significantly differ between groups.

The current study, while utilizing a systematic random sampling grid and the nucleator to estimate somal volume, deviated from stereologic methods based on unbiased 
principles in several key ways. First, we did not uniformly randomly sample the full rostral-caudal extent of BA42. Thus, our findings are limited to that portion of BA42 from which we sampled and may not generalize to BA42 as a whole. Second, as a prerequisite for definitive identification both of a specific cortical sublayer and of pyramidal neurons, only sections where BA42 was cut perpendicular to the pial surface were sampled. Consequently, our observations might not generalize to regions of BA42 tangential to the coronal plane of section. Third, for comparability to our prior study in BA9 (Pierri et al, 2001), conducted using an isotropic nucleator at a time when the vertical nucleator application was not available in our laboratory, the current study again used the isotropic nucleator despite not having obtained isotropic uniform random sections. The extent to which this choice might have induced bias in the measurement of neuronal volume has not been subjected to empirical test, although one study found no detectable bias when measuring neuronal nuclear volume in mice (Schmitz et al, 1999). Brain tissue volume can change substantially during tissue fixation, storage, and histological processing. It is unknown if, or to what extent, individual neuronal volumes are similarly altered during fixation, storage, and subsequent processing. The validity of neuronal volume comparisons is dependent on the assumption that neuronal volumes in the schizophrenic and comparison subjects were not differentially altered by these processes. Finally, it should be noted that reduced neuron somal volume in subjects with schizophrenia has now been reported in specific cortical layers within selected brain regions in several studies (Benes et al, 1991; Arnold et al, 1995; Rajkowska et al, 1998; Pierri et al, 2001), despite a diversity of regions examined, sampling paradigms, and measurement techniques. It is difficult to postulate a systematic methodologic bias that would account for this pattern of results, although future studies based on unbiased principles would increase confidence in this conclusion.

In keeping with the structural observations described above, multiple studies have found subjects with schizophrenia to demonstrate deficits in the precision of auditory sensory memory (Strous et al, 1995; Javitt et al, 1997, 2000a; Rabinowicz et al, 2000; Wible et al, 2001). These deficits include early, or preattentive, elements of auditory cortical sensory processing (Javitt et al, 2000b). Although the circuits underlying these phenomena are incompletely understood, they appear to be dependent on intact auditory association cortex (Dewson III et al, 1970; Cowey and Dewson, 1972; Iverson and Mishkin, 1973; Cowey and Weiskrantz, 1976; Colombo et al, 1990; Wible et al, 2001). In monkey, the auditory association cortex lateral to the primary auditory cortex (grossly corresponding to human region BA42) has been further subdivided on the basis of parvalbumin immunostaining into lateral belt and parabelt regions (Jones et al, 1995a; Kosaki et al, 1997; Hackett et al, 1998a). Auditory tone sensory input diverges into two parallel processing streams projecting from the medial geniculate nucleus to both primary auditory cortex and lateral belt cortex (Molinari et al, 1995; Hackett et al, 1998b; Kaas et al, 1999). These two regions receive little direct input, however, from heteromodal thalamic nuclei such as the pulvinar or medial dorsal nucleus (Morel et al, 1993;
Pandya et al, 1994; Hackett et al, 1998b), and limited direct projections from heteromodal prefrontal cortex (Romanski et al, 1999; Hackett et al, 2001). In contrast, the auditory parabelt has substantial inputs from pulvinar and from prefrontal cortex (Pandya et al, 1994; Hackett et al, 1998b, 2001; Romanski et al, 1999). Auditory parabelt in turn demonstrates reciprocal connections with lateral belt cortex, but not primary auditory cortex (Morel and Kaas, 1992; Hackett et al, 1998a). This linkage of the lateral belt and parabelt cortex may provide the gateway through which the primary auditory sensory stream converges with polysensory and prefrontal input. Since deep layer 3 pyramidal cells of the lateral belt and parabelt in part furnish the projections that reciprocally connect these two regions (Pandya and Sanides, 1973; Pandya and Rosene, 1993; Jones et al, 1995b), an abnormality in these neurons is powerfully positioned to disrupt this convergence. Consistent with this interpretation, in a study examining the effects of bilateral ablation of the parabelt cortex on deficits in auditory sensory memory in nonhuman primates, the severity of deficit was correlated with the extent of the additional lesion of the lateral belt cortex (Colombo et al, 1996).

In view of the circuitry described above, the finding of a high degree of correlation between reduced deep layer 3 pyramidal somal volumes in BA42 and in BA9 deserves special comment. We had previously hypothesized that the reduced pyramidal cell somal volume, and associated reductions in dendrite and dendritic spine markers, in dPFC in subjects with schizophrenia may have resulted from deafferentation owing to reduced numbers of neurons in the mediodorsal thalamic nucleus (Pakkenberg, 1990; Glantz and Lewis, 2000; Young et al, 2000; Popken et al, 2000; Pierri et al, 2001; Byne et al, 2002). Although there is limited thalamic input from the mediodorsal thalamic nucleus to the auditory association cortex (Morel et al, 1993; Pandya et al, 1994; Hackett et al, 1998b), recent evidence suggests that the pulvinar may also be affected in schizophrenia (Byne et al, 2001, 2002). Thus, the common alterations in deep layer 3 pyramidal neurons in BA42 and BA9 may reflect a shared disturbance of nuclei that furnish their thalamic inputs. Alternatively, the correlation in somal volume reductions between BA9 and BA42 could reflect impairments in the reciprocal corticocortical projections connecting these regions that arise, at least in part, from layer 3 pyramidal neurons. These reciprocal connections are much more extensive in the auditory parabelt than in the lateral belt cortex (Romanski et al, 1999; Hackett et al, 2001), raising the possibility that this hypothesis could be tested after first identifying in human brain the regions corresponding to the auditory lateral belt and parabelt.

Adding to the substantial in vivo evidence of structural and functional impairments of auditory association cortex in subjects with schizophrenia, we found reduced pyramidal cell somal volume in deep layer 3 of BA42. Further studies are needed to determine whether the reduction in mean pyramidal cell somal volume results from a change in cell size, or from an altered number of large or small cells. Similarly, the interpretation of the current findings, and the design of future investigations of abnormalities of auditory association cortex, will be enriched by initial studies to 
identify and map the auditory lateral belt and parabelt in human brain.

\section{ACKNOWLEDGMENTS}

This work was supported by USPHS Grant MH 45156 and a grant from the Stanley Foundation. We thank Mr Colin Stebbins for assistance with the figures. We gratefully acknowledge the efforts of the research staff of the Program on Translational Neuroscience and the Center for Neuroscience of Mental Disorders at the University of Pittsburgh.

\section{REFERENCES}

Arnold SE, Franz BR, Gur RC, Gur RE, Shapiro RM, Moberg PJ et al (1995). Smaller neuron size in schizophrenia in hippocampal subfields that mediate cortical-hippocampal interactions. Am J Psychiatry 152: 738-748.

Barta PE, Pearlson GD, Brill II LB, Royall R, McGilchrist IK, Pulver $\mathrm{AE}$ et al (1997). Planum temporale asymmetry reversal in schizophrenia: replication and relationship to gray matter abnormalities. Am J Psychiatry 154: 661-667.

Benes FM, Sorensen I, Bird ED (1991). Reduced neuronal size in posterior hippocampus of schizophrenic patients. Schizophr Bull 17: $597-608$.

Byne W, Buchsbaum MS, Kemether E, Hazlett EA, Shinwari A, Mitropoulou V et al (2001). Magnetic resonance imaging of the thalamic mediodorsal nucleus and pulvinar in schizophrenia and schizotypal personality disorder. Arch Gen Psychiatry 58: 133-140.

Byne W, Buchsbaum MS, Mattiace LA, Hazlett EA, Kemether E, Elhakem SL et al (2002). Postmortem assessment of thalamic nuclear volumes in subjects with schizophrenia. Am J Psychiatry 159: $59-65$.

Colombo M, D'Amato MR, Rodman HR, Gross C (1990). Auditory association cortex lesions impair auditory short-term memory in monkeys. Science 247: 336-338.

Colombo M, Rodman HR, Gross CG (1996). The effects of superior temporal cortex lesions on the processing and retention of auditory information in monkeys (cebus apella). J Neurosci 16: 4501-4517.

Cowey A, Dewson JH (1972). Effects of unilateral ablation of superior temporal cortex on auditory sequence discrimination in macca mulatta. Neuropsychologia 10: 279-289.

Cowey A, Weiskrantz L (1976). Auditory sequence discrimination in macca mulatta: the role of the superior temporal cortex. Neuropsychologia 14: 1-10.

Dewson III JH, Cowey A, Weiskrantz L (1970). Disruptions of auditory sequence discrimination by unilateral and bilateral cortical ablations of superior temporal gyrus in the monkey. Exp Neurol 28: 529-548.

Falkai P, Bogerts B, Schneider T, Greve B, Pfeiffer U, Pilz K et al (1995). Disturbed planum temporale asymmetry in schizophrenia. A quantitative post-mortem study. Schizophr Res 14: 161176.

Galaburda A, Sanides F (1980). Cytoarchitectonic organization of the human auditory cortex. J Comp Neurol 190: 597-610.

Garey LJ (1999). Description of individual brain maps. In: Garey LJ (ed). Brodmann's 'Localisation in the Cerebral Cortex'. Imperial College Press: Covenant Garden, London. pp 122-125.

Glantz LA, Lewis DA (2000). Decreased dendritic spine density on prefrontal cortical pyramidal neurons in schizophrenia. Arch Gen Psychiatry 57: 65-73.
Goldstein JM, Goodman JM, Seidman LJ, Kennedy DN, Makris N, Lee $\mathrm{H}$ et al (1999). Cortical abnormalities in schizophrenia identified by structural magnetic resonance imaging. Arch Gen Psychiatry 56: 537-547.

Gundersen HJG (1988). The nucleator. J Microscopy 151: 3-21.

Gundersen HJG, Bagger P, Bendtsen TF, Evans SM, Korbo L, Marcussen $\mathrm{N}$ et al (1988). The new stereological tools: dissector, fractionator, nucleator and point sampled intercepts and their use in pathological research and diagnosis. Acta Pathol Microbiol Immunol Scand 96: 857-881.

Hackett TA, Stepniewska I, Kaas JH (1998a). Subdivisions of auditory cortex and ipsilateral cortical connections of the parabelt auditory cortex in macaque Monkeys. J Comp Neurol 394: 475-495.

Hackett TA, Stepniewska I, Kaas JH (1998b). Thalamocortical connections of the parablet auditory cortex in macaque monkeys. J Comp Neurol 400: 271-286.

Hackett TA, Stepniewska I, Kaas JH (2001). Prefrontal connections of the parabelt auditory cortex in macaque monkeys. Brain Res 817: $45-48$

Heffner HE (1997). The role of macaque auditory cortex in sound localization. Acta Otolaryngol (Stockh) 532(S): 22-27.

Heffner HE, Heffner RS (1989). Effect of restricted cortical lesions on absolute thresholds and aphasia-like deficits in Japanese macaques. Behav Neurosci 103: 158-169.

Highley JR, McDonald B, Walker MA, Esiri MM, Crow TJ (1999). Schizophrenia and temporal lobe asymmetry: a post-mortem stereological study of tissue volume. Br J Psychiatry 175: 127134.

Hirayasu Y, McCarley RW, Salisbury DF, Tanaka S, Kwon JS, Frumin $M$ et al (2001). Planum temporale and heschl gyrus volume reduction in schizophrenia. Arch Gen Psychiatry 57: 692-699.

Hirayasu Y, Shenton ME, Salisbury DF, Dickey CC, Fischer IA, Mazzoni P et al (1998). Lower left temporal lobe MRI volumes in patients with first-episode schizophrenia compared with psychotic patients with first-episode affective disorder and normal subjects. Am J Psychiatry 155: 1384-1391.

Iverson SD, Mishkin M (1973). Comparison of superior temporal and inferior prefrontal lesions on auditory and non-auditory tasks in rhesus monkeys. Brain Res 55: 355-367.

Jacobs B, Driscoll L, Schall M (1997). Life-span dendritic and spine changes in areas 10 and 18 of human cortex: a guantitative golgi study. I Comp Neurol 386: 661-680.

Javitt DC, Shelley AM, Ritter W (2000a). Associated deficits in mismatch negativity generation and tone matching in schizophrenia. Clin Neurophysiol 111: 1733-1737.

Javitt DC, Shelley AM, Silipo G, Lieberman AR (2000b). Deficits in auditory and visual context-dependent processing in schizophrenia. Arch Gen Psychiatry 57: 1131-1137.

Javitt DC, Strous RD, Grochowski S, Ritter W, Cowan N (1997). Impaired precision, but normal retention, of auditory sensory ('echoic') memory information in schizophrenia. J Abnorm Psychology 106: 315-324.

Johnson NL, Kotz S, Balakrishnan N (1994). Continuous Univariate Distributions. Wiley \& Sons: New York, NY.

Jones EG, Dell'Anna ME, Molinari M, Rausell E, Hashikawa T (1995a). Subdivisions of macaque monkey auditory cortex revealed by calcium-binding protein immunoreactivity. J Comp Neurol 362: 153-170.

Jones EG, Dell'Anna ME, Rausell ME, Hashikawa T (1995b). Subdivisions of macaque monkey auditory cortex revealed by calcium binding protein immunoreactivity. J Comp Neurol 362: 153-170.

Kaas JH, Hackett TA, Tramo MJ (1999). Auditory processing in primate cerebral cortex. Curr Opin Neurobiol 9: 164-170.

Kosaki H, Hashikawa T, He J, Jones EG (1997). Tonotopic organization of auditory cortical fields delineated by parvalbu- 
min immunoreactivity in macaque monkeys. J Comp Neurol 386: 304-316.

Lidow MS, Song ZM, Castner SA, Allen PB, Greeengard P, Goldman-Rakic PS (2001). Antipsychotic treatment induces alterations in dendrite-and-spine associated proteins in dopamine rich areas of the primate cerebral cortex. Biol Psychiatry 49: $1-12$

Littell RC, Milliken GA, Stroup WW, Wolfinger RD (1996). SAS System for Mixed Models. SAS Institute Inc.: Cary, NC.

Mathalon DH, Sullivan EV, Lim KO, Pfefferbaum A (2001). Progressive brain volume changes and the clinical course of schizophrenia in men. Arch Gen Psychiatry 58: 148-157.

Mathew VM, Gruzelier JH, Liddle PF (1991). Lateral asymmetries in auditory acuity distinguish hallucination from nonhallucinating schizophrenic patients. Psychiatry Res 46: 127-138.

McCarley RW, Wible CG, Frumin M, Hirayasu Y, Levitt JJ, Fischer IA et al (1999). MRI anatomy of schizophrenia. Biol Psychiatry 45: 1099-1119.

Mendis-Handagama SMLC, Ewing LL (1990). Sources of error in the estimation of Leydig cell numbers in control and atrophied mammalian testes. J Micros 159: 73-82.

Mirra SS, Heyman A, McKeel D, Sumi SM, Crain BJ, Brownlee LM et al (1991). The consortium to establish a registry for Alzheimer's disease (CERAD). Part II. Standardization of the neuropathologic assessment of Alzheimer's disease. Neurology 41: 479-486.

Molinari M, Dell'Anna ME, Rausell E, Leggio MG, Hashikawa T, Jones EG (1995). Auditory thalamocortical pathways defined in monkeys by calcium-binding protein immunoreactivity. J Comp Neurol 362: 171-194.

Morel A, Garraghty PE, Kaas JH (1993). Tonotopic organization, architectonic fields, and connections of auditory cortex in macaque monkeys. J Comp Neurol 335: 437-459.

Morel A, Kaas JH (1992). Subdivisions and connections of auditory cortex in owl monkeys. J Comp Neurol 318: 27-63.

Neter J, Kutner MH, Nachtsheim CJ, Wasserman W (1996). Applied Linear Statistical Models. Irwin: Chicago, IL.

Pakkenberg B (1990). Pronounced reduction of total neuron number in mediodorsal thalamic nucleus and nucleus accumbens in schizophrenics. Arch Gen Psychiatry 47: 1023-1028.

Pakkenberg B (1993). Total nerve cell number in neocortex in chronic schizophrenics and controls estimated using optical dissectors. Biol Psychiatry 34: 768-772.

Pandya DN, Rosene DL (1993). Laminar termination patterns of thalamic, callosal, and association afferents in the primary auditory area of the rhesus monkey. Exp Neurol 119: 220-234.

Pandya DN, Rosene DL, Doolittle AM (1994). Corticothalamic connections of auditory-related areas of the temporal lobe in the rhesus monkey. J Comp Neurol 345: 447-471.

Pandya DN, Sanides F (1973). Architectonic parcellation of the temporal operculum in rhesus monkey and its projection pattern. Z Anat Entwicklungsgesch 139: 127-161.

Pierri JN, Edgar CL, Auh S, Sampson A, Lewis DA (2001). Decreased somal size of deep layer 3 pyramidal neurons in the prefrontal cortex in schizophrenia. Arch Gen Psychiatry 58: 466473.

Popken GJ, Bunney Jr WE, Potkin SG, Jones EG (2000). Subnucleus-specific loss of neurons in medial thalmus of schizophrenics. Proc Natl Acad Sci 97: 9276-9280.

Rabinowicz EF, Silipo G, Goldman R, Javitt DC (2000). Auditory sensory dysfunction in schizophrenia. imprecision or distractibility? Arch Gen Psychiatry 57: 1149-1155.

Rajkowska G, Selemon LD, Goldman-Rakic PS (1998). Neuronal and glial somal size in the prefrontal cortex: a postmortem morphometric study of schizophrenia and Huntington disease. Arch Gen Psychiatry 55: 215-224.

Romanski LM, Bates JF, Goldman-Rakic PS (1999). Auditory belt and parabelt projections to the prefrontal cortex in the rhesus monkey. J Comp Neurol 403: 141-157.

Sanfilpo M, Lafargue T, Rusinek H, Arena L, Loneragan C, Lautin A et al (2000). Volumetric measure of the frontal and temporal lobe regions in schizophrenia. Arch Gen Psychiatry 57: 471-480.

Schmitz C, Schuster D, Nissen P, Korr H (1999). No difference between estimated mean nuclear volumes of various types of neurons in the mouse brain obtained on either isotropic uniform random sections or conventional frontal or sagittal sections. $J$ Neurosci Methods 88: 71-82.

Shenton ME, Kikinis R, Jolesz FA, Pollak SD, LeMay M, Wible CG et al (1992). Abnormalities of the left temporal lobe and thought disorder in schizophrenia. $N$ Engl J Med 327: 604-612.

Sigmundsson T, Suckling J, Williams SCR, Bullmore ET, Greenwood KE, Fukuda R et al (2001). Structural abnormalities in frontal, temporal and limbic regions and interconnecting white matter tracts in schizophrenic patients with prominent negative symptoms. Am J Psychiatry 158: 234-243.

Strous RD, Cowan N, Ritter W, Javitt DC (1995). Auditory sensory ('echoic') memory dysfunction in schizophrenia. Am J Psychiatry 152: 1517-1519.

Sullivan EV, Mathalon DH, Lim KO, Marsh L, Pfefferbaum A (1998). Patterns of regional cortical dysmorphology distinguishing schizophrenia and chronic alcoholism. Biol Psychiatry 43: 118-131.

Vogeley K, Hobson T, Schneider-Axmann T, Honer WG, Bogerts B, Falkai P (1998). Compartmental volumetry of the superior temporal gyrus reveals sex differences in schizophrenia - a postmortem study. Schizophr Res 31: 83-87.

Wible CG, Kubicki M, Yoo SS, Kacher DF, Salisbury DF, Anderson MC et al (2001). A functional magnetic resonance imaging study of auditory mismatch in schizophrenia. Am J Psychiatry 158: 938-943.

Young KA, Manaye KF, Liang CL, Hicks PB, German DC (2000). Reduced number of mediodorsal and anterior thalamic neurons in schizophrenia. Biol Psychiatry 47: 944-953.

Zaidel DW, Esiri MM, Harrison PJ (1997). Size, shape, and orientation of neurons in the left and right hippocampus: investigation of normal asymmetries and alterations in schizophrenia. Am J Psychiatry 154: 812-818. 